\title{
A Novel Method for Handling Vehicle Occlusion in Visual Traffic Surveillance
}

\author{
Clement C. C. Pang*, William W. L. Lam and Nelson H. C. Yung \\ Department of Electrical and Electronic Engineering, \\ The University of Hong Kong, Hong Kong SAR
}

\begin{abstract}
This paper presents a novel algorithm for handling occlusion in visual traffic surveillance (VTS) by geometrically splitting the model that has been fitted onto the composite binary vehicle mask of two occluded vehicles. The proposed algorithm consists of a critical points detection step, a critical points clustering step and a model partition step using the vanishing point of the road. The critical points detection step detects the major critical points on the contour of the binary vehicle mask. The critical points clustering step selects the best critical points among the detected critical points as the reference points for the model partition. The model partition step partitions the model by exploiting the information of the vanishing point of the road and the selected critical points. The proposed algorithm was tested on a number of real traffic image sequences, and has demonstrated that it can successfully partition the model that has been fitted onto two occluded vehicles. To evaluate the accuracy, the dimensions of each individual vehicle are estimated based on the partitioned model. The estimation accuracies in vehicle width, length and height are $95.5 \%, 93.4 \%$ and $97.7 \%$ respectively.
\end{abstract}

Keywords: Visual traffic surveillance, occlusion, model-based tracking, model partitioning

\section{INTRODUCTION}

Visual traffic surveillance (VTS) has been actively investigated in the past ten years due to its high potential of becoming one of the dominant components of the Intelligent Transportation System (ITS) [7]. As the name implies, visual traffic surveillance has a powerful feature that it allows the system to visualize vehicles on the road, thus enabling traffic scene analysis, such as traffic conditions assessment, speed estimation as well as queue length measurement in which traditional surveillance systems could not do [2]. However, unlike traditional surveillance systems, such as inductive loops and laser sensors, the performance of a VTS system deteriorates when there is vehicle occlusion in the traffic image sequence. As a result, algorithms for occlusion handling have to be employed in order to make the surveillance system usable, since vehicle occlusion occurs frequently in traffic image sequence.

Algorithms for occlusion handling for indoor object tracking has been reported in the past [1][3][5]. However, there is a lack of focused research in outdoor visual traffic surveillance. Although, there are several groups of researchers who have attempted to tackle the problem as a subset of their larger goal [2][6][7], none of them have really addressed this problem decidedly. This has motivated us to focus our investigation on this problem alone.

In this paper, we propose a novel algorithm for handling occlusion by splitting the model that has been fitted onto the composite binary vehicle mask of two occluded vehicles. The assumption is that when two vehicles are occluded as seen in an image sequence, by means of segmentation techniques, the composite mask (inclusive of two vehicles) can be extracted. If a model is fitted to this composite mask, the result is an erroneous model which can be highly misleading. Our objective is to sub-divide or split the model geometrically so that the sub-divided models describe the individual vehicles, where dimensions of individual vehicles can be extracted accurately. The rest of this paper is organized as follow. A brief problem analysis is given in the next section. Following that, the proposed methodology is introduced in section 3. Experimental results and discussion is given in section 4, and the conclusion can be found in section 5 .

*ccpang@eee.hku.hk; phone 852-2857-8414. 


\section{PROBLEM ANALYSIS}

In model-based vehicle tracking, vehicle masks are usually extracted from the image before the model is fitted onto the vehicle in order to simplify the process of fitting. The method known as "background subtraction" is commonly employed for the extraction of vehicle masks. After the subtraction, refinement techniques such as closing or convex hull transformation may be applied to the vehicle mask to remove spurious noise as well as holes and concavity in the mask. At this stage, a deformable model may be fitted onto the binary mask based on the geometric shape of the mask (Figure 1). Dimensions of the vehicle are then extracted based on the size of the fitted model [8].

In the case when two vehicles are occluding each other, their binary masks overlap with each other (Figure 2), and as a result, one large model will be fitted onto the composite binary masks instead of two separate ones. This obviously will cause the algorithm to extract the wrong dimension parameters from such a model. Moreover, the recovery of the vehicle trajectory can also badly affected, since there might be matching ambiguities of vehicles between frames [7]. In order to solve this problem, the composite model must be partitioned into two separate ones so that each model fits onto one vehicle only. The key question is how this can be done and algorithmically accurate.
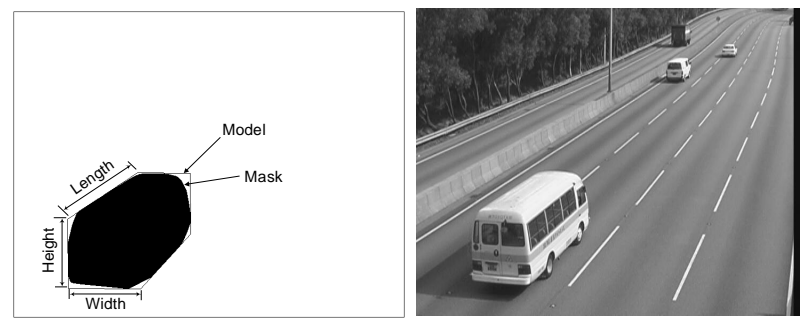

Figure 1: The model fitted onto the binary vehicle mask
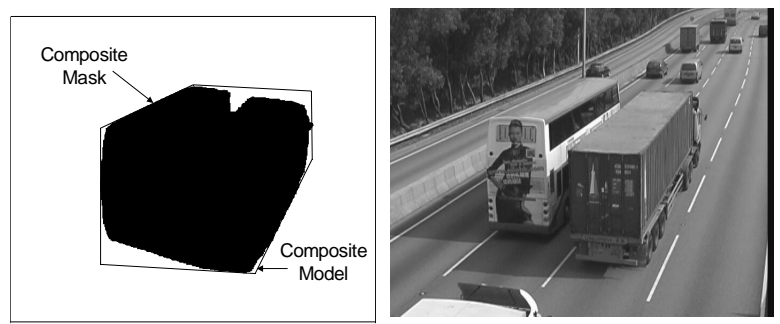

Figure 2: The model fitted onto the overlapped binary vehicle mask of two occluded vehicles

An efficient way to partition the model is to exploit the geometrical properties of the model. This could mean the identification of critical points on the boundary of the composite vehicle mask and the determination of the line of partition. From Figure 3, we can observe that every vector which is in the positive Y direction (ie., line $\boldsymbol{V}_{2} \boldsymbol{V}_{\boldsymbol{l}}$, line $\boldsymbol{V}_{4} \boldsymbol{V}_{5}$, line $\boldsymbol{V}_{7} \boldsymbol{V}_{6}$, where $\boldsymbol{V}_{\boldsymbol{i}}$ denote the vertices) in the 3D space, when projected back to the 2D image plane, will converge to a vanishing point $p$. By exploiting this point in the 2D space, with the aid of the set of critical points on the binary vehicle mask (which is defined as the points on the contour of the composite vehicle mask where there are major directional changes), we should be able to partition the composite model into smaller models. A detail explanation of the method of critical points extraction as well as the partitioning approach will be given in the next section.

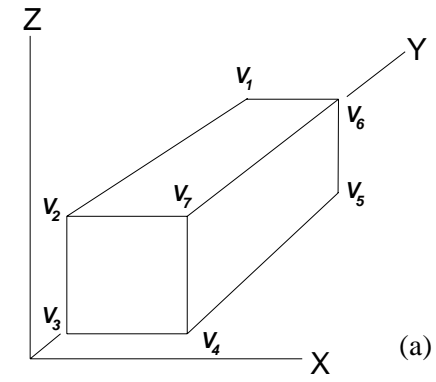

Figure 3: 3D cuboid in (a) 3D world coordinates;

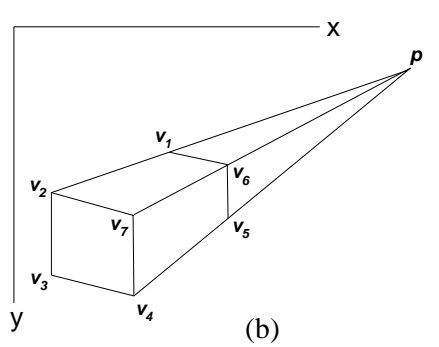

(b) 2D perspective view.

\subsection{Critical Points Extraction}

\section{PROPOSED METHOD}

Before partitioning the model, critical points on the contour outline of the composite binary vehicle mask have to be identified (Figure 4(a)). To achieve this, a signature curve of the outline contour is first constructed (Figure 4(b)). The 
signature curve $\rho(i)$ is obtained by computing the Euclidean distances between the centre of gravity $(\boldsymbol{g})$ of the composite mask and every data sample on the contour $(\boldsymbol{x}(\boldsymbol{i}))$. The resulted curve is defined as

$$
\rho(i)=\|\boldsymbol{x}(i)-\boldsymbol{g}\|^{2} \quad i=1, \ldots, N .
$$

where $N$ is the number of points on the contour, and centre of gravity is defined as the mean coordinate of all the samples on the contour, as follow:

$$
\boldsymbol{g}=\frac{1}{N} \sum_{i=1}^{N} \boldsymbol{x}(i)
$$

The reason for choosing the above parameters for computing the signature curve is that they are isotropic. From a real $\boldsymbol{x}(\boldsymbol{i})$, we can observe that the curve is inherently noisy. The noise of the signature curve is the result of sudden changes on the contour due to vehicle parts such as wheels, side mirror and bumper ends. As they are, these points are not the most essential points for determining the partition lines, and they therefore should be filtered out. If the signature curve is not smoothed, then too many critical points will be detected, which will introduce ambiguities when choosing critical points for partition. In our study, we have employed a lowpass filter with cutoff frequency $\omega_{c}=0.1 \pi$ radians. This choice of filter parameters can eliminate most of the rapid changes on the signature curve, without filtering out the essential features on the contour. The signature curve after lowpass filtering is shown in Figure 4(c). This curve is then subjected to local extrema detection by locating the zero crossing points of the first derivative of the curve. The resultant points are labeled as $\boldsymbol{c}_{\boldsymbol{i}}$ for $i=1, \ldots, 10$, which correspond to the critical points as shown in Figure 4(a). They represent a set of points in determining the partition line of the model.

The number of resultant critical points on the contour after the filtering process is usually around ten for two occluded vehicles. However, in some occasions, there might be more than ten critical points detected. The reason is that the corners on the vehicles are not sharp enough, which leads to multiple points detected around the brunt corners. In order to solve this problem, the critical points around the brunt corners are clustered by evaluating their tangential slopes, and the one with tangential slope closest to zero will be chosen as the real critical point.

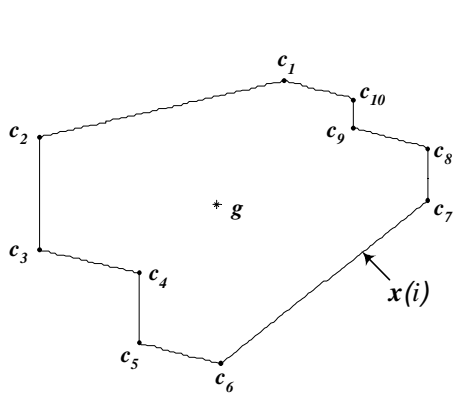

(a)

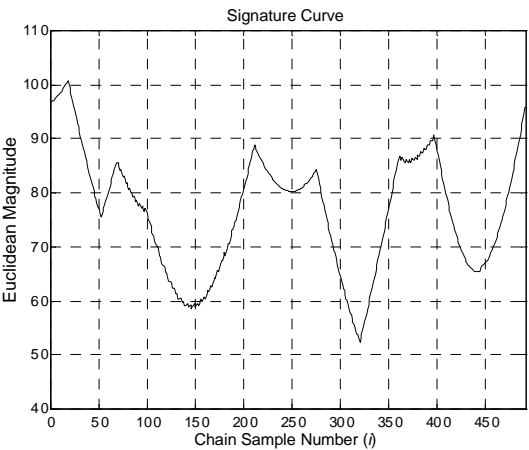

(b)

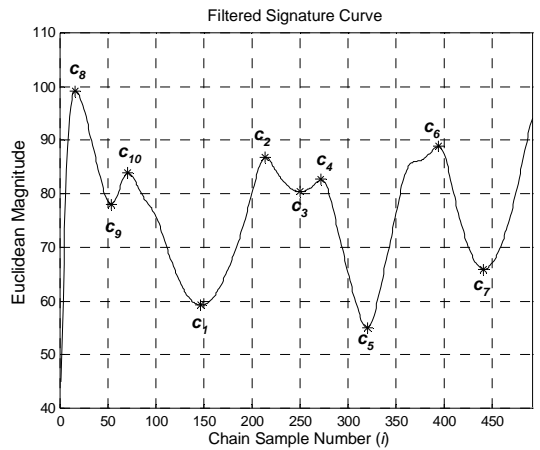

(c)

Figure 4: $\quad \begin{array}{ll}\text { (a) Syntactic contour of binary vehicle mask } & \text { (b) Real signature curve }\end{array}$ (c) Signature curve subjected to low pass filtering and critical points identified

\subsection{Model partition}

With the critical points $\boldsymbol{C}=\left\{\boldsymbol{c}_{j}\right\}$ for $j=1, \ldots, K$ and the vanishing point $\boldsymbol{p}$ in hand, the composite model can be partitioned by the steps introduced in this section. As mentioned in the preceding section, $K$ is usually around 10 to denote the critical points of two vehicles under occlusion. Assume that model $X$ is fitted onto the composite mask of two occluded vehicles, namely, vehicle $A$ and $B$ by performing the procedures that were mentioned in Section 2 (Figure 5). Moreover, assume that both vehicles are traveling in the same direction. The vertices of model $X$ can be labeled as $v_{x}=\left\{v_{x 1}, v_{x 2}, v_{x 3}, v_{x 4}, v_{x 5}, v_{x 6}\right\}$. By transforming the vertices of model $X$ to the $3 \mathrm{D}$ world coordinates, the dimensions of the model can be extracted as follows:

$$
\text { width }=\left|V_{x 3}-V_{x 4}\right| ; \quad \text { length }=\left|V_{x 4}-V_{x 5}\right| ; \quad \text { height }=\left|V_{x 2}-V_{x 3}\right|,
$$


where $V_{\mathrm{x}}=\left\{\boldsymbol{V}_{x 1}, \boldsymbol{V}_{x 2}, \boldsymbol{V}_{x 3}, \boldsymbol{V}_{x 4}, \boldsymbol{V}_{x 5}, \boldsymbol{V}_{x \boldsymbol{}}\right\}$ denotes the vertices of model $X$ in the $3 \mathrm{D}$ world coordinates. Our task is to partition $v_{x}$ into two separate models, namely, $v_{a}=\left\{\boldsymbol{v}_{a 1}, \boldsymbol{v}_{a 2}, \boldsymbol{v}_{a 3}, \boldsymbol{v}_{a 4}, \boldsymbol{v}_{a 5}, \boldsymbol{v}_{a \boldsymbol{6}}\right\}$ and $v_{b}=\left\{\boldsymbol{v}_{\boldsymbol{b} 1}, \boldsymbol{v}_{\boldsymbol{b} 2}, \boldsymbol{v}_{\boldsymbol{b} 3}, \boldsymbol{v}_{\boldsymbol{b} 4}, \boldsymbol{v}_{\boldsymbol{b} 5}, \boldsymbol{v}_{b 6}\right\}$, so that $v_{a}$ and $v_{b}$ describe the dimensions of vehicles $a$ and $b$ respectively. From Figure 5, we can observe that points $\boldsymbol{v}_{a 1}, \boldsymbol{v}_{a 2}, \boldsymbol{v}_{\boldsymbol{b} 4}$ and $v_{b 5}$ correspond to $v_{x 1}, v_{x 2}, v_{x 4}$ and $v_{x 5}$ respectively and are already known from $v_{x}$. What we have to do is then to determine points $v_{a 3}, v_{a 4}, v_{a 5}, v_{a b}$ for vehicle $A$, and points $v_{b 1}, v_{b 2}, v_{b 3}, v_{b 6}$ for vehicle $B$.

By the steps mentioned in Section 3.1, we can observe from Figure 5 that $c_{1}, c_{2}, c_{6}, c_{7}$ correspond to model points $v_{x 1}$, $v_{x 2}, v_{x 4}, v_{x 5}$ respectively. Therefore, we only need to exploit $c_{3}$, $c_{5}, c_{8}, c_{10}$ in this partitioning step. They correspond to $v_{a 3}, v_{b 3}$, $v_{b 6}, v_{a b}$ respectively.

Based on these four points, we can construct part of $\boldsymbol{v}_{\boldsymbol{a}}$ by traversing line $\boldsymbol{v}_{x 3} \boldsymbol{v}_{x 4}$ upwards and line $\boldsymbol{v}_{x 5} \boldsymbol{v}_{x 6}$ leftwards until they fit vehicle $a$ (Figure 5). Similarly, we can construct part of $v_{b}$ by traversing line $v_{x 2} v_{x 3}$ rightwards, and line $v_{x 6} v_{x 1}$ downwards until they fit vehicle $b$. The intersections of these lines with the fitted model $v_{x}$ define points $v_{a 3}, v_{a b}, v_{b 3}$ and $v_{b 6}$.

By defining a vanishing point $\boldsymbol{p}$, we use the following steps to determine points $\boldsymbol{v}_{\boldsymbol{a} 4}, \boldsymbol{v}_{\boldsymbol{a}}$ for vehicle $A$, and points $\boldsymbol{v}_{\boldsymbol{b} 1}$, $v_{b 2}$ for vehicle $B$ :

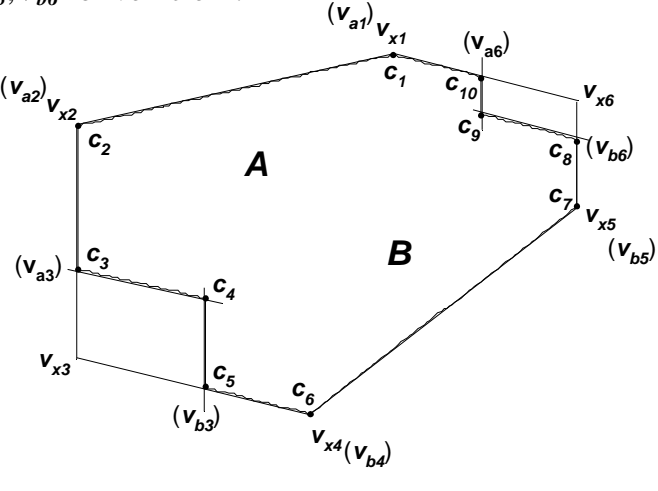

Figure 5: A model fitted onto two occluded vehicles

\section{Vehicle $A$ (Figure 6)}

1) Extend line $v_{x 2} v_{x 1}$ and line $\boldsymbol{v}_{x 4} \boldsymbol{v}_{x 5}$ until they intersect at point $\boldsymbol{p}$ (Figure 6(a))

2) Connect point $\boldsymbol{p}$ and point $\boldsymbol{v}_{a b}$, and extend the line.

3) Extend line $\boldsymbol{v}_{\boldsymbol{a}} \boldsymbol{v}_{\boldsymbol{a} 1}$ along plane $\mathbf{p} \boldsymbol{v}_{\boldsymbol{a}} \boldsymbol{v}_{a 1}$ until intersect with point $\boldsymbol{v}_{\boldsymbol{a} 2}$. New corner on the extended plane is now labeled $v_{a 7}$ (Figure 6(b)).

4) Project line $\boldsymbol{v}_{\boldsymbol{a} 2} \boldsymbol{v}_{\boldsymbol{a} 3}$ along the plane $\boldsymbol{v}_{\boldsymbol{a} 2} \boldsymbol{v}_{\boldsymbol{a} 3} \boldsymbol{v}_{\boldsymbol{a}}$ until intersect with point $\boldsymbol{v}_{\boldsymbol{a} 7}$. The intersection between the new line and the horizontal line passing through $v_{a 3}$ is now labeled as $\boldsymbol{v}_{a 4}$ (Figure 6(c)).

5) Connect point $\boldsymbol{p}$ and $\boldsymbol{v}_{a 4}$. An intersection point $\boldsymbol{v}_{a 5}$ is formed (Figure 6(d)).
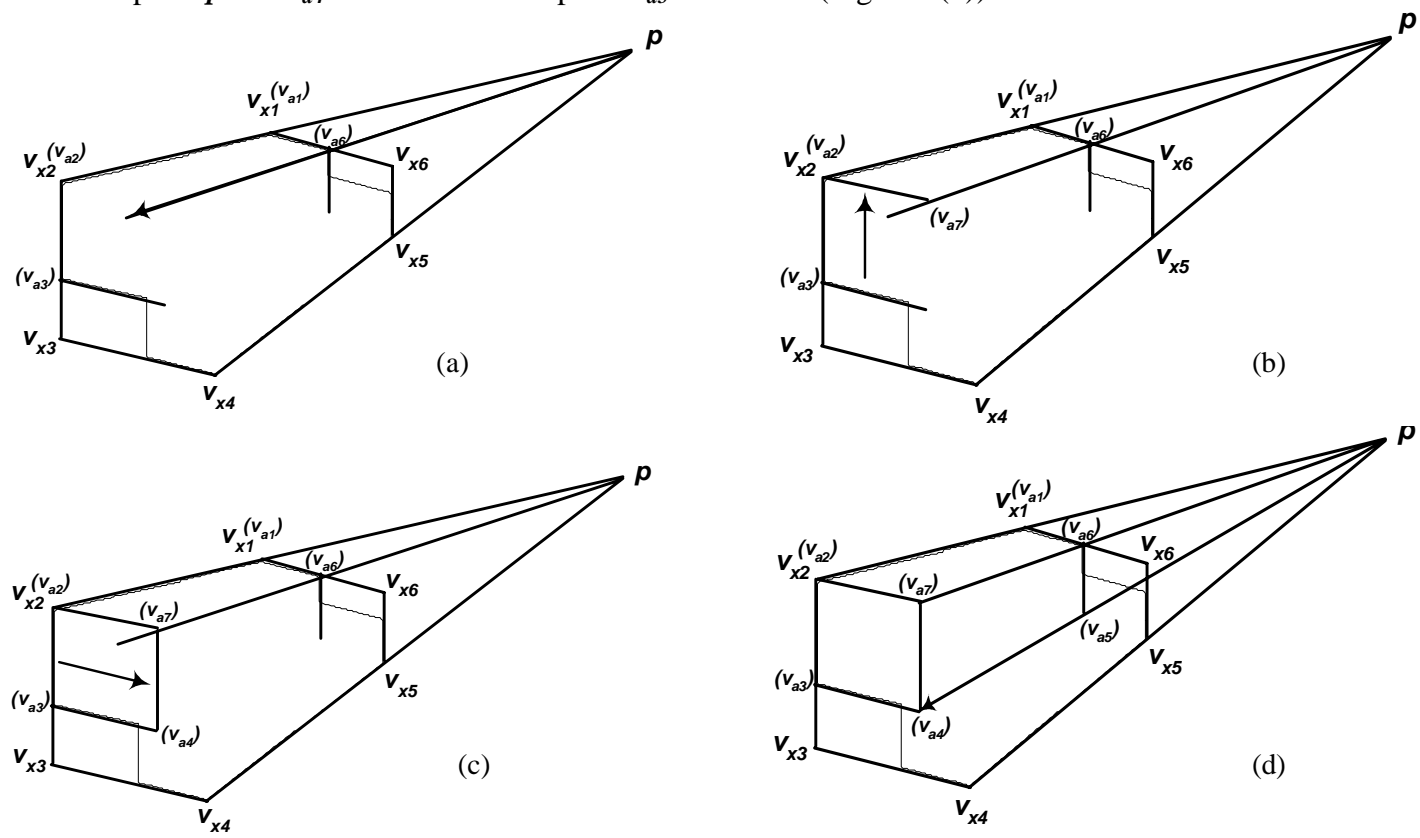

Figure 6: Steps to partition vehicle $A$ 


\section{Vehicle $B$ (Figure 7)}

1) Extend line $\boldsymbol{v}_{x 2} \boldsymbol{v}_{x 1}$ and line $\boldsymbol{v}_{x 4} \boldsymbol{v}_{x 5}$ until they intersect at point $\boldsymbol{p}$ (Figure 7(a)).

2) Connect point $p$ and $v_{b 6}$, and extend the line.

3) Extend line $v_{b 5} v_{b \boldsymbol{b}}$ along plane $\boldsymbol{p} \boldsymbol{v}_{\boldsymbol{b} 5} \boldsymbol{v}_{\boldsymbol{b} \boldsymbol{6}}$ until intersect with point $\boldsymbol{v}_{\boldsymbol{b} 4}$. New corner on the extended plane is now labeled $v_{b 7}$ (Figure 7(b)).

4) Project line $v_{b 3} v_{b 4}$ upwards until intersect with point $v_{b 7}$. The intersection between the new line and the vertical line passing through $\boldsymbol{v}_{\boldsymbol{b} 3}$ is now labeled as $\boldsymbol{v}_{\boldsymbol{b} 2}$ (Figure 7(c)).

5) Connect point $\boldsymbol{p}$ and $\boldsymbol{v}_{\boldsymbol{b} 2}$. An intersection point $\boldsymbol{v}_{\boldsymbol{b} 1}$ is formed (Figure 7(d)).

By the above steps, the model can be partitioned into two separate but complete models $v_{a}$ and $v_{b}$.
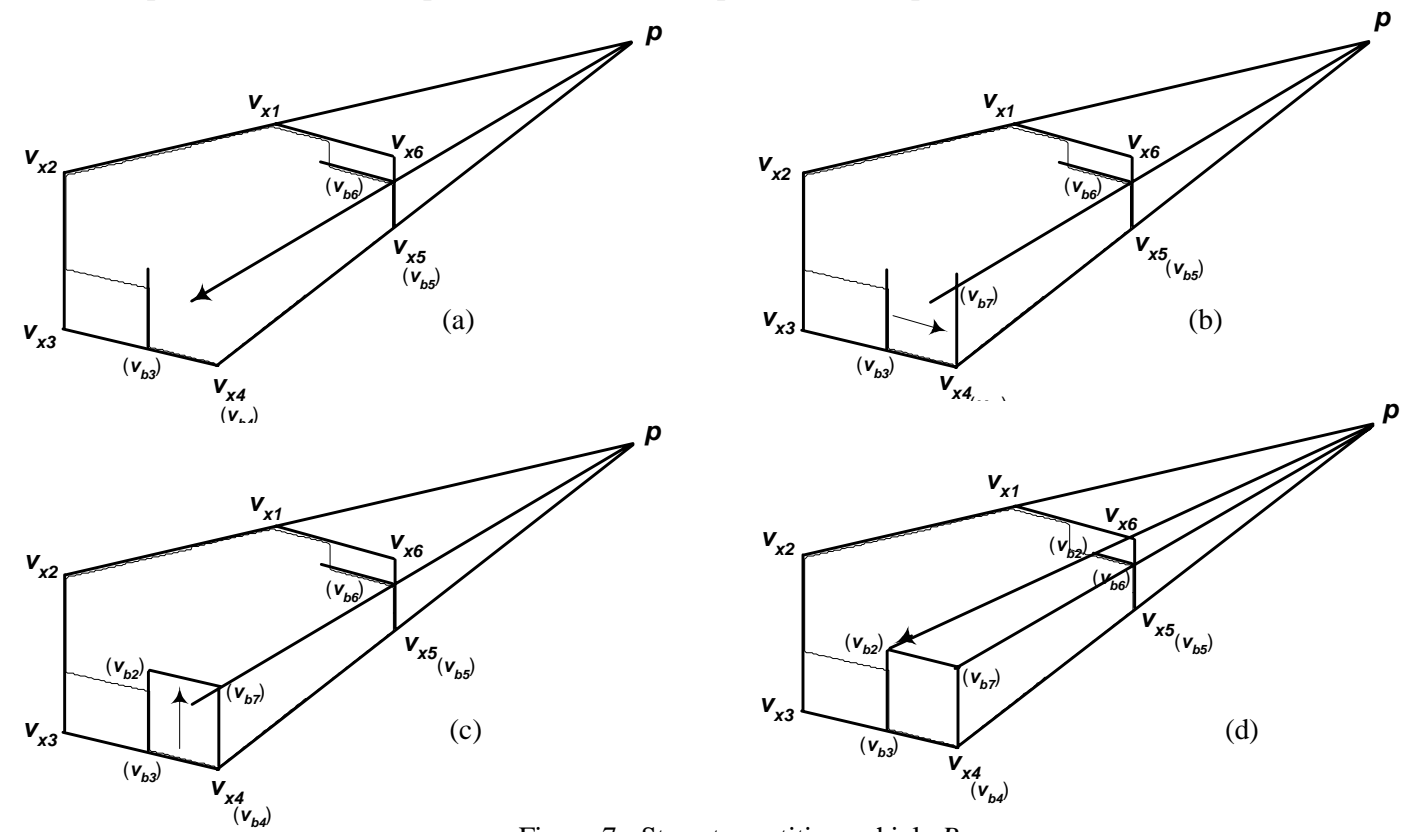

Figure 7: Steps to partition vehicle $B$

\section{EXPERIMENTAL RESULTS AND DISCUSSION}

The newly proposed algorithm was tested on two sets of real traffic images that were taken on a busy highway where occlusions occur frequently. Both sets of images were taken during daytime where the shadow effects are prominent. The shadow removal algorithm proposed by [Fun01] was employed to remove the cast shadow in the vehicles.

The first set of image contains a double-decker bus that was partially occluded by an articulated-truck (Figure 8(a)). The signature curve of the contour is first computed by Eqt.(1) (Figure 8(c)). The signature curve is then subjected to lowpass filtering, and the result of the filtering is depicted in Figure 8(d). The critical points are then detected from the filtered signature curve (Figure 8(e)). Figure 9(f) depicts all the detected critical points on the contour. The detected critical points are then clustered (Figure $8(\mathrm{~g})$ ). It is noted that the number of critical points is reduced as shown in Figure $8(\mathrm{~h})$. By exploiting these critical points as well as the vanishing point $\boldsymbol{p}$ of the road, the model was partitioned into two portions by using the algorithm as introduced in Section 3.2 (Figure 8(i)).

The individual vehicle after partition is shown in Figure $8(\mathrm{j})$ and $8(\mathrm{k})$. We observe that the double-decker bus was accurately segmented from the image. However, the segmentation is slightly biased to the left for the articulatedtruck. This deviation is due to the fact that the right edge of the partitioned model (ie. line $\boldsymbol{v}_{\boldsymbol{b} \boldsymbol{s}} \boldsymbol{v}_{\boldsymbol{b} \boldsymbol{b}}$ ), was slightly deviated from the vehicle. This deviation is caused by the side mirror on the right hand side of the truck. The deviation of line $v_{b 5} v_{b 6}$ has also caused line $v_{b 1} v_{b 2}$ to be slightly offset from the edge of the truck. 
The second set of images contains two mini-vans in which one of them is occluding another. The generation of the signature curve, low-pass filtering, critical points detected, clustering and model partition can be seen in Figure 9(c)-(i) respectively. From Figure 9(j) and (k), we observe that the segmentations of both vans are quite accurate this time. Moreover, unlike the articulated-truck in the previous set, the side mirror of the right van does not cause too much problem of model offset. This is because the side mirror of the mini-van is aligned nearer to the vehicle body than the one in the articulated-truck. Therefore the effect on segmentation error is less prominent than the articulatedtruck. The problem of side mirror is usually less pronounced in small vehicles than in large vehicles.

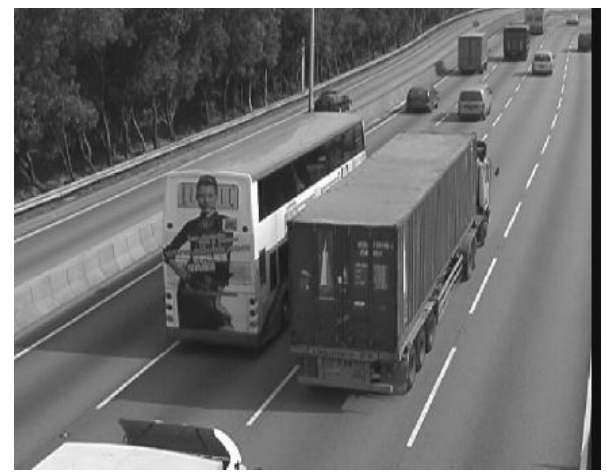

(a)

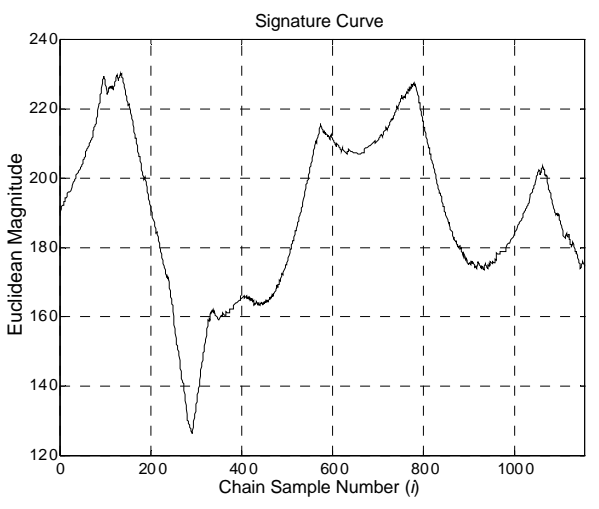

(c)

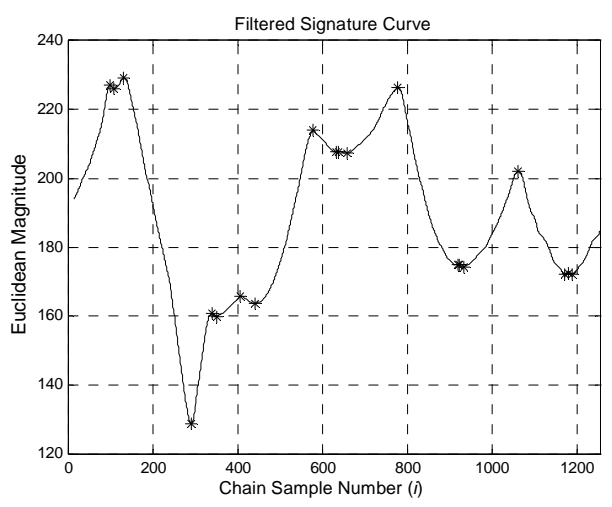

(e)

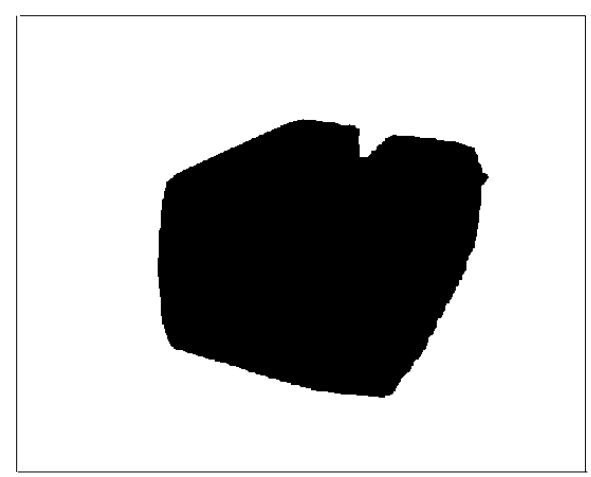

(b)

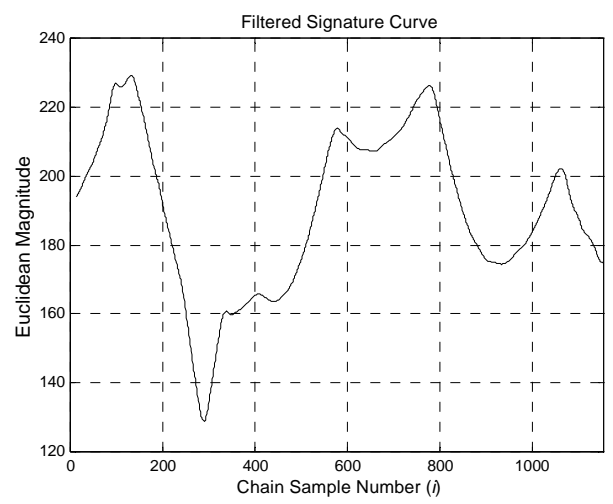

(d)

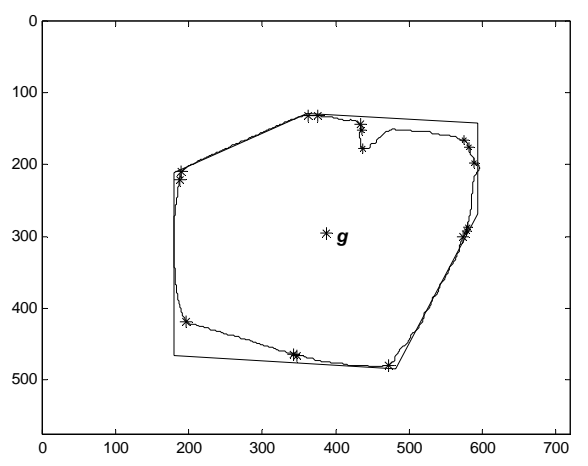

(f) 


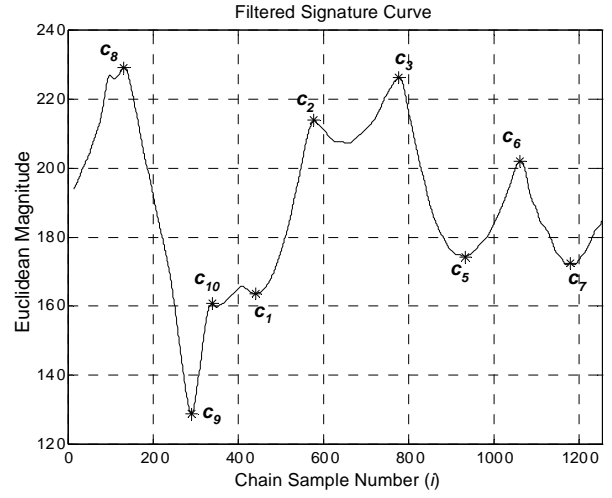

(g)

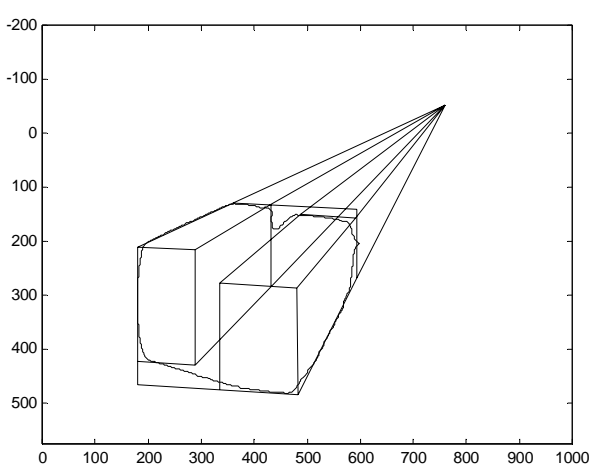

(i)

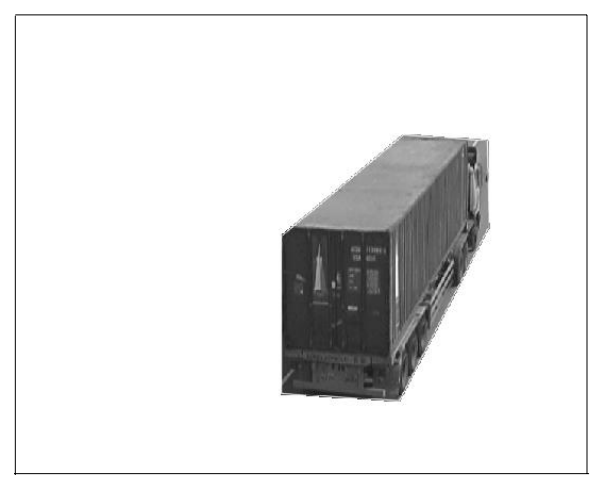

(k)

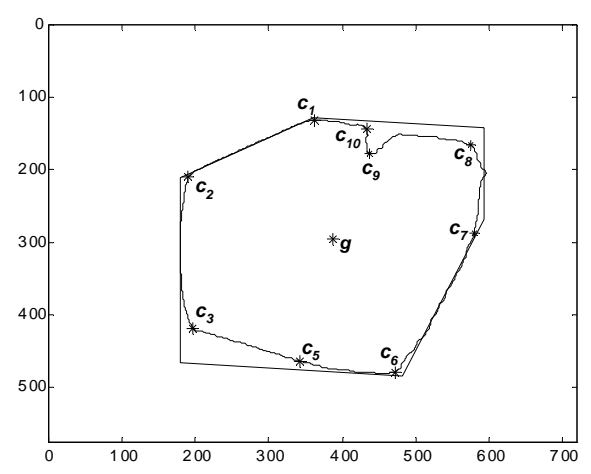

(h)

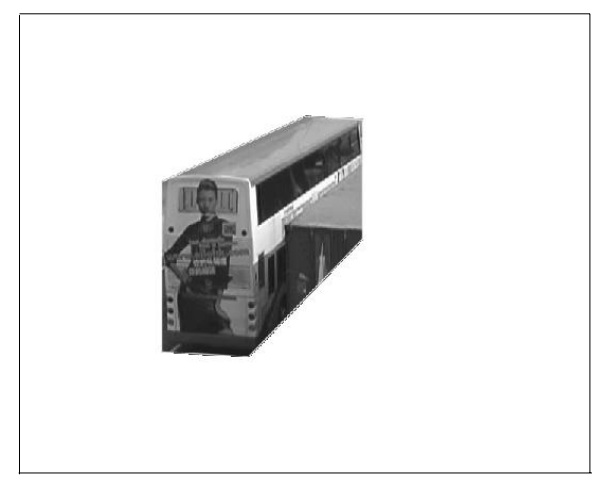

(j)

Figure 8: The model partition of a bus and an articulatedtruck
(a) Original diagram
(b) Binary vehicle mask
(c) Signature curve
(d) Filtered signature curve
(e) \& (f) Detected critical points
(g) \& (h) Clustered critical points
(i) Partitioned lines of the model
(j) Partitioned model of the bus
(k) Partitioned model of the truck 


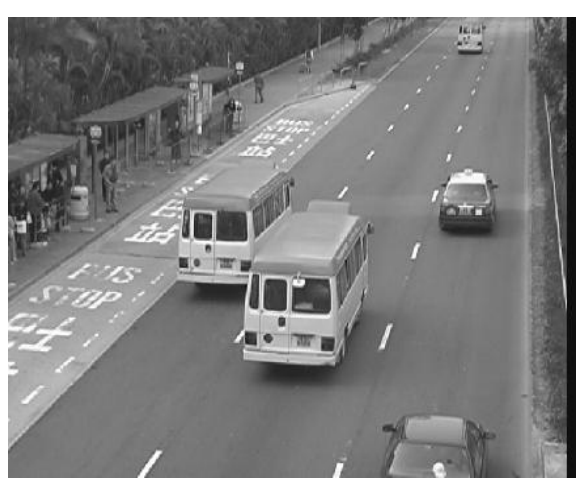

(a)

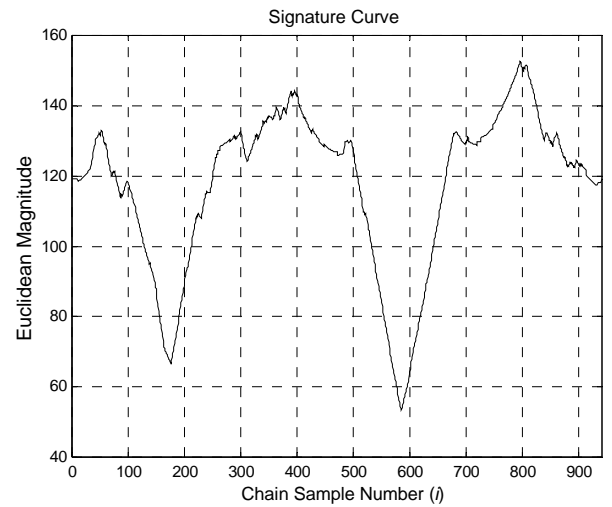

(c)

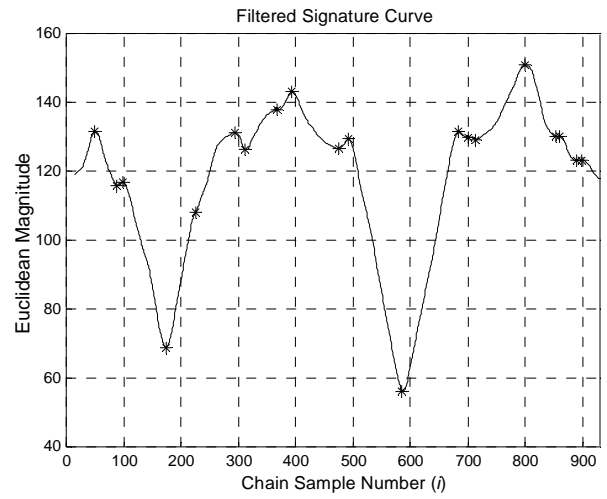

(e)

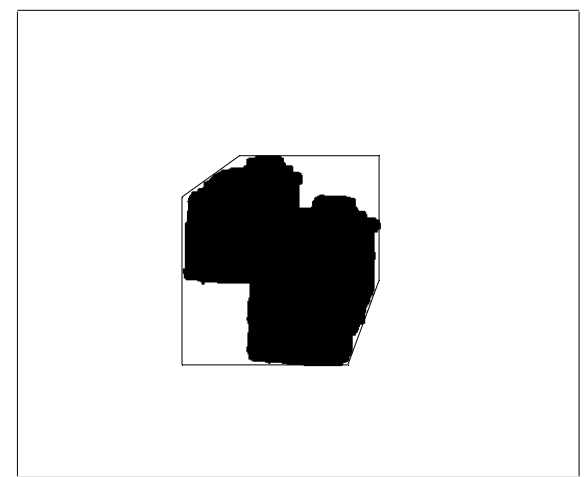

(b)

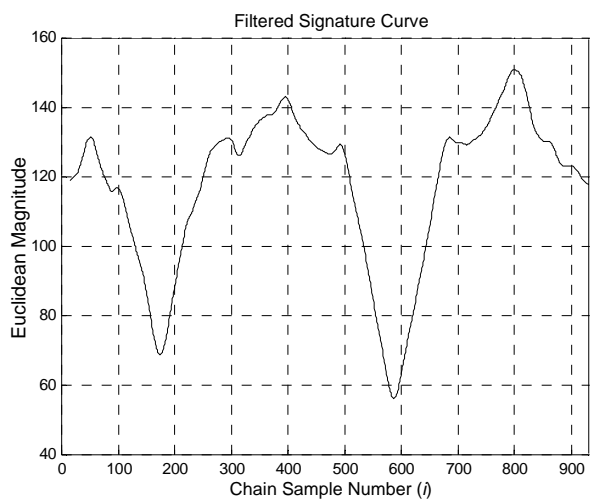

(d)

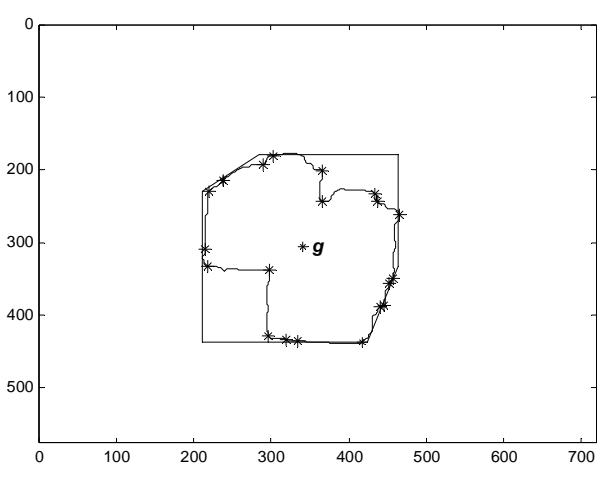

(f) 


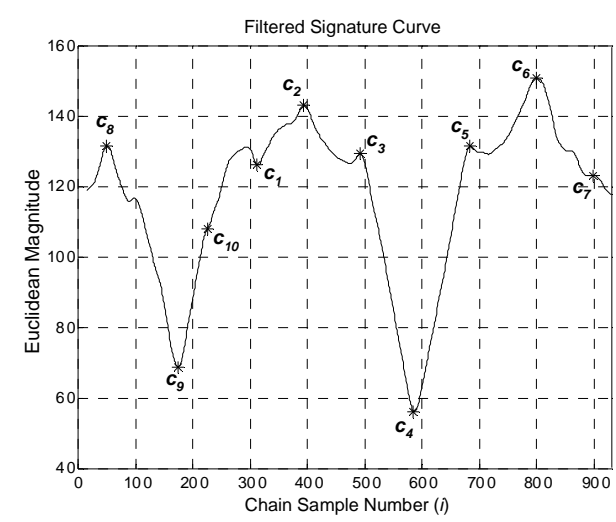

(g)

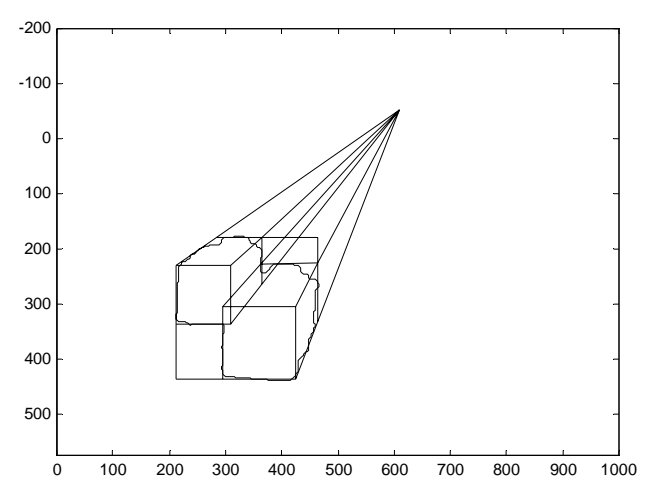

(i)

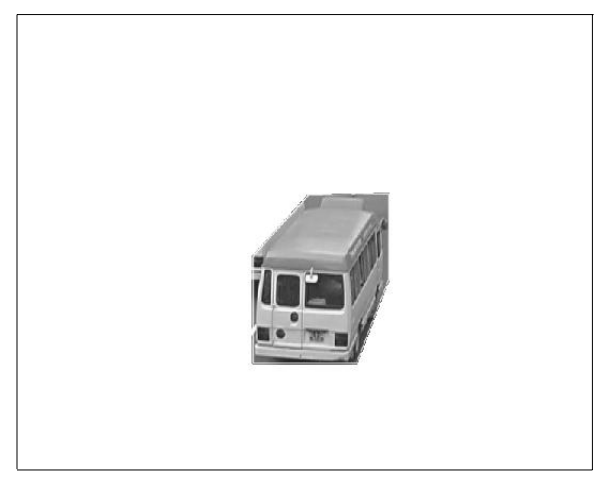

(k)

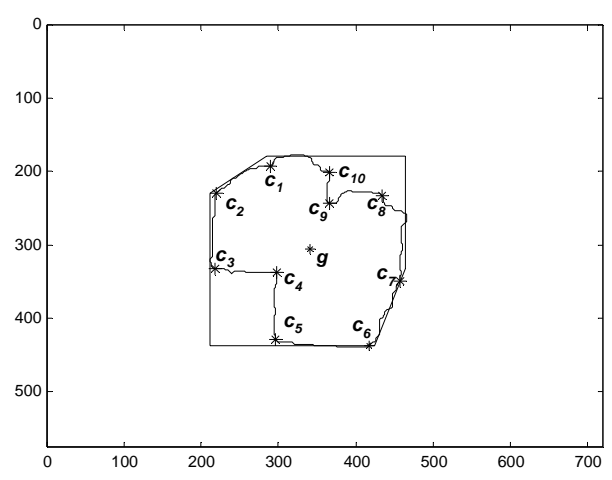

(h)

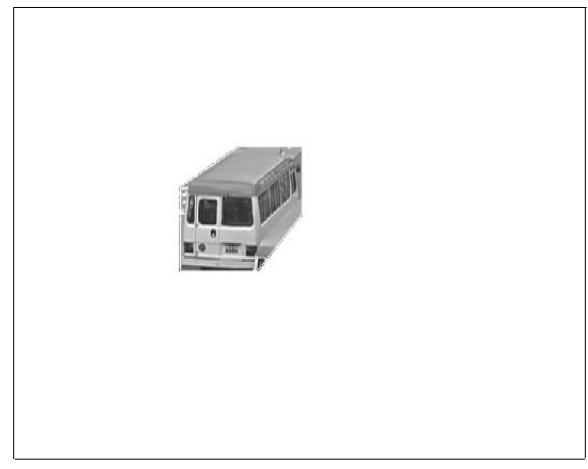

(j)

Figure 9: The model partition of two occluded mini-vans
(a) Original diagram
(c) Signature curve
(e) \& (f) Detected critical points
(g) \& (h) Clustered critical points
(i) Partitioned lines of the model
(j) Partitioned model of the left van
(k) Partitioned model of the right van

(b) Binary vehicle mask

(d) Filtered signature curve 
To evaluate the accuracy of the proposed algorithm, the dimensions of the vehicles in the test images were extracted from the partitioned model. The estimated values are then compared with the actual dimension of the vehicle published by the manufacturer. The dimension of the double-decker bus is tabulated in Table 1 . The error in width and height is around $4.5 \%$, while the error in length is slightly higher than the width and height (around $8 \%$ ). This is probably due to the fact that the edge of model $A$ which describes the length of the double-decker bus (ie., line $\boldsymbol{v}_{a 4} \boldsymbol{v}_{\boldsymbol{a} 5}$ ) does not fit the vehicle perfectly.

Table 2 tabulates the estimated dimensions of the articulated-truck in Figure 8. From the table, we can observe that the height of the vehicle is accurately estimated (error $<1.5 \%$ ). The error of width is around $7 \%$, while the length has a relatively higher error (around 10\%). The large error in the width and length is due to the side mirror on the right hand side of the vehicle which caused the offset of the model during model fitting. As mentioned before, the side mirror of large vehicles usually causes significant error in dimension estimation.

The error in the estimated dimensions of the two mini-vans is relatively smaller than that of the double-decker bus and the articulated-truck, as shown in Table 3 and 4. The reason for the accurate estimation is that the binary mask of the two occluded mini-vans has clear and distinguishable features, so that the critical points for partition can be located accurately from the outline contour. Moreover, the side mirrors of the mini-vans are relatively closer to the vehicle body than those of the articulated-trucks, which cause less offset of the model.

\begin{tabular}{|l|c|c|c|}
\hline \multicolumn{1}{|c|}{$(\mathrm{mm})$} & Width & Length & Height \\
\hline Actual & 2500 & 11880 & 4760 \\
\hline Estimated & 2622 & 12871 & 4970 \\
\hline Error & $4.88 \%$ & $8.34 \%$ & $4.41 \%$ \\
\hline
\end{tabular}

Table 1 Extracted dimensions of the double-decked bus (Figure 8)

\begin{tabular}{|l|c|c|c|}
\hline \multicolumn{1}{|c|}{$(\mathrm{mm})$} & Width & Length & Height \\
\hline Actual & 2400 & 12000 & 2550 \\
\hline Estimated & 2225 & 13208 & 2582 \\
\hline Error & $7.29 \%$ & $10.07 \%$ & $1.25 \%$ \\
\hline
\end{tabular}

Table 2 Extracted dimensions of the articulated truck (Figure 8)

\begin{tabular}{|l|c|c|c|}
\hline \multicolumn{1}{|c|}{$(\mathrm{mm})$} & Width & Length & Height \\
\hline Actual & 2025 & 6255 & 2585 \\
\hline Estimated & 1938 & 6575 & 2649 \\
\hline Error & $4.30 \%$ & $5.12 \%$ & $2.48 \%$ \\
\hline
\end{tabular}

Table 3 Extracted dimensions of the left van (Figure 9)

\begin{tabular}{|l|c|c|c|}
\hline \multicolumn{1}{|c|}{$(\mathrm{mm})$} & Width & Length & Height \\
\hline Actual & 2025 & 6255 & 2585 \\
\hline Estimated & 2059 & 6424 & 2616 \\
\hline Error & $1.68 \%$ & $2.70 \%$ & $1.20 \%$ \\
\hline
\end{tabular}

Table 4 Extracted dimensions of the right van (Figure 9)

In general, height estimation is the most accurate and robust, whilst width accuracy is determined by the side mirror, and the length has the largest variation and least accuracy.

\section{CONCLUSION}

In conclusion, the occlusion-handling algorithm based on partitioning the model as presented in this paper is an effective way for handling vehicle occlusion. The merit of the proposed method is that each occluded vehicles can be modeled and measured individually with good accuracy. The proposed algorithm has been tested on real world traffic images, and the average estimation accuracy in vehicle width is $95.5 \%$, whereas the accuracies in vehicle length and 
height are $93.4 \%$ and $97.7 \%$ respectively. The drawback of the algorithm is that the performance is hindered by the side-mirror of the vehicles, especially for large vehicles such as articulated-trucks, and solutions should be found in order to relief this effect. Future development of this method will be focused on developing a model for describing the occlusion of multiple vehicles (both occluded horizontally and vertically) so that this model can applied to solve occlusions of multiple vehicles reliably.

\section{REFERENCES}

[1] Benlamri R., "Curved shapes construction for object recognition”. Proceedings of the Geometric Modeling and Processing, pp. 197-204, 2002.

[2] Beymer D. and Malik K., "Tracking vehicles in congested traffic". Proceedings of the IEEE Intelligent Vehicles Symposium, pp. 130-135, 1996.

[3] Castellani U., Livatino S. and Fisher R. B., "Improving environment modeling by edge occlusion surface completion". Proceedings: First International Symposium on 3D Data Processing Visualization and Transmission, pp. 672-675, 2002.

[4] Fung G.S.K., Yung N.H.C., Pang G.K.H. and Lai A.H.S., "Effective Moving Cast Shadow Detection for Monocular Color Traffic Image Sequences". Optical Engineering- The Journal of SPIE, Vol. 41(6), pp. 14251440, 2002. (ISSN 0091-3286)

[5] Grimson W.E.L., Object recognition by computer: the role of geometric constraints, MIT Press, 1990.

[6] Koller D., Daniididis K. and Nagel H. H., "Model-based object tracking in monocular image sequences of road traffic scenes”. International Journal of Computer Vision, vol. 10, no. 3, pp. 257-281, 1993.

[7] Lai A.H.S, An Effective Methodology for Visual Traffic Surveillance, PhD Dissertation, The University of Hong Kong, Jan 2000.

[8] Lai A.H.S, Fung G.S.K. and Yung, N.H.C., "Vehicle type classification from visual-based dimension estimation”. Proceedings: IEEE Intelligent Transportation Systems Conference, pp. 201-206, 2001.

[9] Yung N.H.C. and Lai A.H.S., "Detection of vehicle occlusion using a generalized deformable model". Proceedings: IEEE International Symposium on Circuits and Systems, vol. 4, pp. 154-157, 1998. 